\title{
P14 INCREASED INFLAMMATORY AND ALTERNATIVE MONOCYTE/MACROPHAGE MARKERS IN PATIENTS WITH HYPERTENSION AND HEART FAILURE WITH PRESERVED EJECTION FRACTION
}

doi:10.1136/heartjnl-2012-303148a.19

${ }^{1} \mathrm{~N}$ Glezeva, ${ }^{* 1,2} \mathrm{P}$ Collier, ${ }^{1} \mathrm{C}$ Watson, ${ }^{2} \mathrm{M}$ Ledwidge, ${ }^{1,2} \mathrm{~K}$ McDonald, ${ }^{1} \mathrm{~J}$ Baugh. ${ }^{1}$ Conway Institute of Biomolecular and Biomedical Research, University College Dublin, Dublin, Ireland; ${ }^{2}$ Heart Failure Unit, St Vincent's University Hospital Healthcare Group, Elm Park, Dublin, Ireland

Inflammation and fibrosis with increased monocyte activation are associated with the progression of left ventricular diastolic dysfunction (LVDD) and heart failure (HF). Three monocyte subclasses exist: classical/inflammatory, intermediate, and alternative/antiinflammatory monocytes. In the tissue monocytes differentiate into inflammatory-M1 or regulatory/pro-fibrotic-M2 macrophages. Alternative monocytes and M2 macrophages have been implicated in the pathogenesis of atherosclerosis/CAD, MI, HF, etc. Since macrophage polarisation may depend on the expression of monocyte markers and cytokine mediators we analysed monocytes from different stage heart disease patients and associated peripheral markers with disease pathogenesis.

Peripheral blood was collected from 83 patients: asymptomatic hypertensive (HTN), LVDD and HF with preserved ejection fraction (HFPEF). Monocytes were purified and stained for CD14, CD16, CD163, CD206. Cytokines in patient plasma were identified by ELISA.

Inflammation in LVDD and HFPEF as compared to HTN was defined by elevated peripheral levels of TNF $\alpha$, IL12, IL6, MCP1. 
These also correlated with peripheral BNP. The M2 cytokines CCL17, CCL18, sCD163 were also increased. The percentage of monocytes was higher in LVDD and HFPEF and overlapped with increased classical and alternative monocytes. Monocyte expression of the M2 receptor CD163 was lower in HFPEF, consistent with high sCD163 and inflammation. Finally, culture of healthy donor monocytes with patient-derived serum stimulated expression of the M2 genes CD206 and IL10.

We therefore suggest that an increased activation and differentiation of monocytes towards regulatory/pro-fibrotic M2 macrophages may occur already in early stage heart disease and that these changes, along with a persistent inflammatory insult, are associated with progression to HFPEF. 